\title{
F-Actin/Myosin-2 Furrow Canals, but not Basal Junctions, are Required for Drosophila Cellularization
}

\author{
Anna Marie Sokac ${ }^{*, * *}$, Margaret Bisher ${ }^{* *}$ and Eric Wieschaus ${ }^{*, * *}$ \\ *Howard Hughes Medical Institute \\ *** Department of Molecular Biology, Princeton University, Princeton, NJ 08544
}

Cellularization of the Drosophila syncytial blastoderm is a modified form of cytokinesis, during which the plasma membrane simultaneously invaginates around each of 6000 cortical nuclei to generate an epithelial sheet of adherent cells that exhibit apical and basal polarity (facing out of and into the embryo, respectively). At early cellularization distinct, cadherin/catenin-based, cell-cell junctions called basal junctions assemble and hold adjacent invaginating plasma membranes together [1]. Basal junctions then move in register with cortical F-actin/myosin-2 furrow canals at invaginating plasma membrane fronts as membrane from internal stores is targeted for insertion just apical to basal junctions [2].

Based on the position of basal junctions during cellularization and the demonstrated roles played by cell-cell junctions in mammalian epithelial cells [3], several molecular functions have been suggested for the basal junctions [4]. First, basal junction assembly may stabilize the initial inward buckling of the plasma membrane between adjacent nuclei. Second, basal junction assembly may induce F-actin polymerization at the basal junction and/or at the immediate furrow canal. Third, basal junctions may limit miscibility between cortical sub-domains, separating the growing lateral plasma membranes from the stable leading F-actin/myosin-2 furrow canals. Fourth, basal junctions may direct polarized membrane insertion by anchoring exocyst complexes.

Using 3D confocal microscopy and TEM we examined the role of basal junctions in cellularization. Embryos deficient for $\beta$-catenin do not form basal junctions, but do initiate and then complete cellularization. That is, the plasma membrane deforms and invaginates around every nucleus, membrane insertion remains polarized and functional furrow canals assemble. Basal junctions are, thus, dispensable for cellularization. Intriguingly though, we use embryos deficient for the basal junction and furrow canal component nullo, which may limit miscibility between cortical subdomains via F-actin, to demonstrate a requirement for intact F-actin/myosin-2 furrow canals in stabilizing initial plasma membrane deformations such that invagination ensues and cellularization proceeds.

\section{References}

[1] Hunter, C., and E. Wieschaus. 2000. J Cell Biol. 150:391-401.

[2] Lecuit, T., and E. Wieschaus. 2000. J. Cell Biol. 150:849-60.

[3] Nelson, J. 2003. Nature. 422:766-774.

[4] Lecuit, T. 2004. J. Cell Sci. 117:3427-3433.

[5] Work funded by a NIH NRSA Post-Doctoral Fellowship to A.M.S. and by HHMI. 

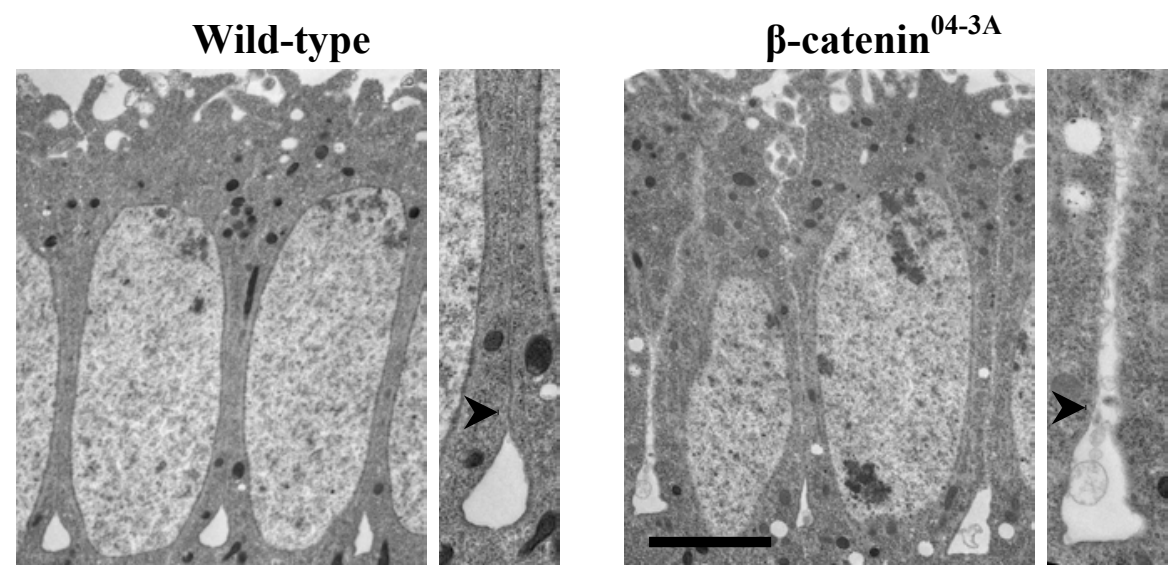

Embryos that are maternally and zygotically deficient for $\beta$-catenin do not form obvious basal junctions. TEM images of wild-type and $\beta$-catenin deficient embryos at low and high magnification (mutant for $\beta$-catenin ${ }^{04-3 \mathrm{~A}}$, a strong hypomorphic allele expressing truncated protein). Notice the gap between adjacent plasma membranes in the $\beta$-catenin mutant at the level where basal junctions should be (arrowhead). Bar $=2 \mu \mathrm{m}$.
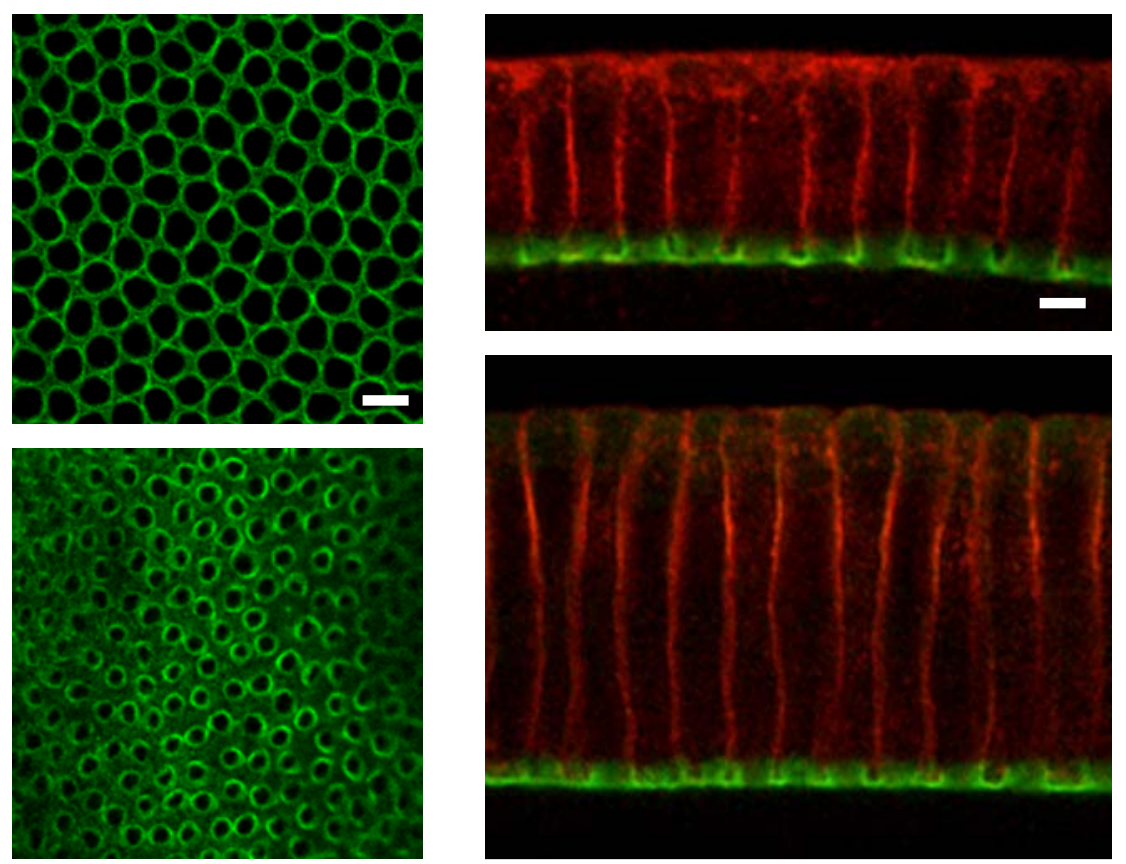

Embryos that are maternally and zygotically deficient for $\beta$-catenin form all furrow canals. Confocal images of furrow canals in $\beta$-catenin mutant embryos. En face images are collected in a single plane at the level of the furrow canals. Neurotactin, a plasma membrane marker, is shown in red; myosin- 2 in green. Notice that in the lower en face image from a latestage cellularizing embryo the furrow canals are constricting laterally, suggesting that not only do furrow canals form, but they are functional in these mutants. Bar $5=\mu \mathrm{m}$. 